\title{
AXIAL DISPLACEMENT DISLOCATIONS FOR THE HOLLOW CONE AND THE HOLLOW SPHERE*
}

\author{
BY \\ J. N. GOODIER AND J. C. WILHOIT, JR. \\ Stanford University
}

Abstract. Simple solutions in closed form are given for the dislocational states of stress in a hollow cone (Fig. 3) or a hollow sphere (Fig. 5) induced by making an axial cut, and imposing a rigid-body displacement of one face of the cut relative to the other, in the axial direction. The problems are solved by adaptations of the Saint-Venant torsion theory, and of J. H. Michell's theory of torsion of non-uniform shafts.

1. Introduction. A classification of elastic dislocations in a hollow cylinder given by V. Volterra [1] in 1907 is shown in Fig. 1, a dislocation being a state of strain induced
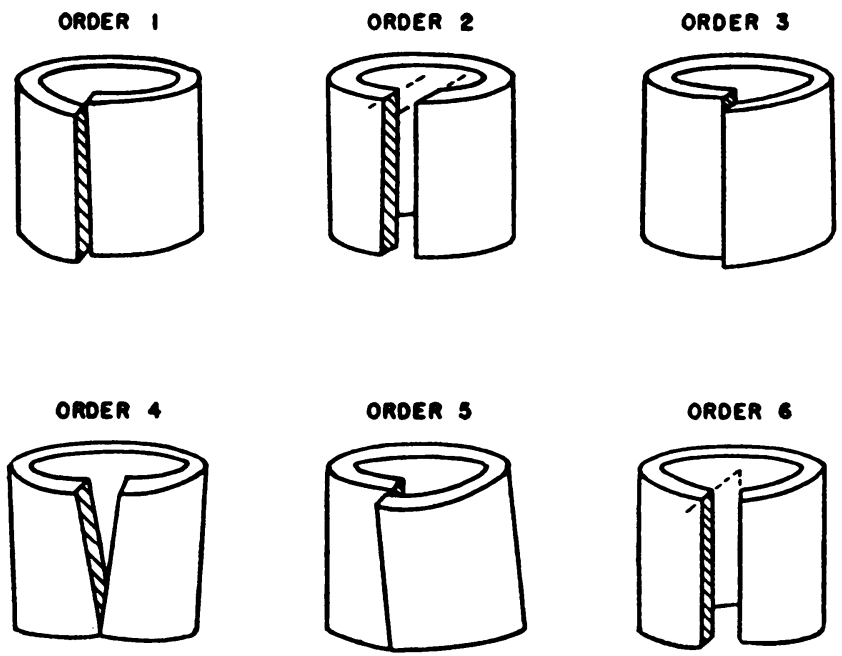

Fig. 1. Volterra's classification of the six dislocations of the hollow cylinder.

by making a cut, imposing relative displacement on the faces, and resealing. The dislocational strain is by definition continuous in the resulting "self-strained" cylinder, at the site of the cut as well as elsewhere, and by a fundamental theorem this requires that the imposed relative displacement be a rigid-body displacement or rotation. We obtain here solutions for the dislocation of order 3 (the relative displacement is an axial translation) in a hollow cone (Fig. 3) and in a hollow sphere (Fig. 5), in simple closed form.

These solutions are derived from the thick-walled cylinder with a dislocation of order 3 , by cutting the hollow cone or the hollow sphere out of it, and finding the effects of removing the tractions on the curved surfaces.

As for the cylinder, the dislocation may be conveniently expressed by an adaptation

*Received August 11, 1954. The paper reports results obtained in the course of research sponsored by the Office of Naval Research at Stanford University (Contract N6-ONR-251, project NR-064-241). 
of the Saint-Venant torsion theory. In terms of Prandtl's stress function $\phi$ the equations** of this theory are

$$
\tau_{x z}=\frac{\partial \phi}{\partial y}, \quad \tau_{y z}=-\frac{\partial \phi}{\partial x}, \quad \nabla^{2} \phi=-2 G \Theta,
$$

$\phi=$ constant on the boundary of the section, and the warping displacement $w$ can be evaluated from the stress-strain relations

$$
\frac{\partial \phi}{\partial y}=G\left(\frac{\partial w}{\partial x}-\theta y\right), \quad-\frac{\partial \phi}{\partial x}=G\left(\frac{\partial w}{\partial y}+\theta x\right) .
$$

The adaptation consists in making the twist $\theta$ zero. In the torsion problem itself this corresponds merely to the unstressed state. The system of equations nevertheless remains capable of expressing certain non-torsional states. Equations (2) become CauchyRiemann equations showing that (with $z=x+i y$, temporarily)

$$
G w+i \phi=f(z) \text {. }
$$

A discontinuity in $w$ of the type required by the dislocation of order 3 is introduced by choosing

$$
f(z)=-i A \log z=A \theta-i A \log r,
$$

$r$ and $\theta$ being polar co-ordinates in the cross-section. Then with $A$ as a real arbitrary constant

$$
G w=A \theta, \quad \phi=-A \log r
$$

and the polar components of shear stress on the section are ( $z$ now being the cylindrical co-ordinate in $r, \theta, z$ )

$$
\tau_{z \theta}=-\frac{\partial \phi}{\partial r}=\frac{A}{r}, \quad \tau_{z r}=0 .
$$

Cylindrical surfaces $r=$ constant are free from stress. The stress is attributable to the discontinuity in $w$

$$
w(r, 2 \pi)-w(r, 0)=2 \pi A / G
$$

at the faces of an axial cut $(\theta=0, \theta=2 \pi)$, but also partly to the torque on the ends $(a<r<b)$

$$
\int_{a}^{b} \tau_{z \theta} 2 \pi r^{2} d r=\pi\left(b^{2}-a^{2}\right) A .
$$

This torque can be removed by superimposing ordinary torsion

$$
\tau_{z \theta}=B r, \quad \tau_{2 r}=0 \quad \text { with } \quad B=-2 A /\left(a^{2}+b^{2}\right),
$$

leaving stress

$$
\tau_{z \theta}=A\left(\frac{1}{r}-\frac{2 r}{a^{2}+b^{2}}\right)
$$

\footnotetext{
**The notation is that of Timoshenko and Goodier, Theory of elasticity, Ch. 11, 1951, except that $\theta$ is used for unit twist instead of $\theta$.
} 
which may be attributed to the dislocational displacement expressed by (7). This nonzero stress prevails on the ends of course as well as throughout the cylinder, but since it has zero resultant its removal would affect only localized end zones.*

2. The hollow cone. In the dislocated cylinder the cylindrical surfaces $r=$ constant are free from traction, but on other surfaces of revolution $f(r, z)=0$ the stress (9) implies ringwise shear loading, and the solution of our problems requires removal of this.

The problem presented by ringwise shear loading on a body of revolution can be formulated as an adaptation of J. H. Michell's theory of torsion of non-uniform circular shafts. In that theory the equations of equilibrium are satisfied by taking

$$
\tau_{r \theta}=-\frac{1}{r^{2}} \frac{\partial \psi}{\partial z} \quad \tau_{z \theta}=\frac{1}{r^{2}} \frac{\partial \psi}{\partial r}
$$

The remaining four stress components are zero. Compatibility of strain requires

$$
\frac{\partial}{\partial r}\left(\frac{1}{r^{3}} \frac{\partial \psi}{\partial r}\right)+\frac{\partial}{\partial z}\left(\frac{1}{r^{3}} \frac{\partial \psi}{\partial z}\right)=0
$$
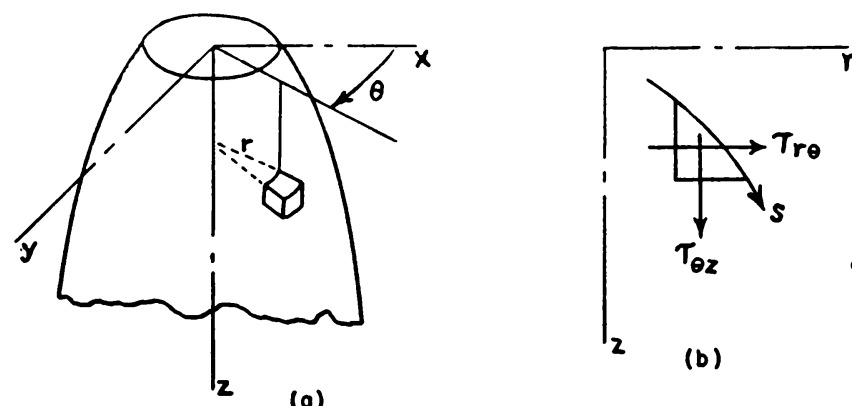

(b)

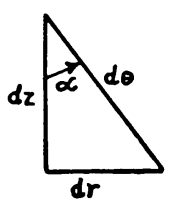

(c)

Fig. 2. Torsional stress in a body of revolution.

In Fig. 2(b) the stresses $\tau_{r \theta}, \tau_{z \theta}$ are shown on an axial section of the solid of revolution in Fig. 2(a), at the boundary $(s)$. The shear stress formed by these two components can be represented as components normal and tangential to the boundary curve. By (10), the normal component $\tau_{\theta n}$ (outward-to the right-in Fig. $2 \mathrm{~h}$ ) is

$$
\tau_{\theta_{n}}=\tau_{r \theta} \cos \alpha-\tau_{\theta z} \sin \alpha=-\frac{1}{r^{2}} \frac{\partial \psi}{\partial s} \text {. }
$$

In the Michell torsion theory this normal component at the boundary is zero, and $\psi$ is constant, because the boundary surface is free. The adaptation to our problem consists simply in retaining (12) with a non-zero prescribed $\tau_{\theta n}$ as a function of $z$ (or $r$ ) on the boundary curve.

*This analysis explains a paradox of thin shell theory. If a thin flat rectangular plate is put under pure shear it becomes a nearly rectangular parallelogram. It can be rolled up into a cylinder, but the joined edges (along a generator) will not quite register. A dislocational axial displacement remains. The shears on the edges remain, and form torques on the ends. It would appear that removal of these torques by simple twisting of the closed cylinder will remove the shear stress altogether, and leave the cylinder dislocated but unstressed. The paradox disappears when variation of stress through the thickness is admitted as in (9). 
The hollow cone (Fig. 3a) is first taken as part of a hollow cylinder in the state expressed by (5), (6), and (7). Spherical co-ordinates $R, \omega, \theta$, indicated in Fig. 3 are used
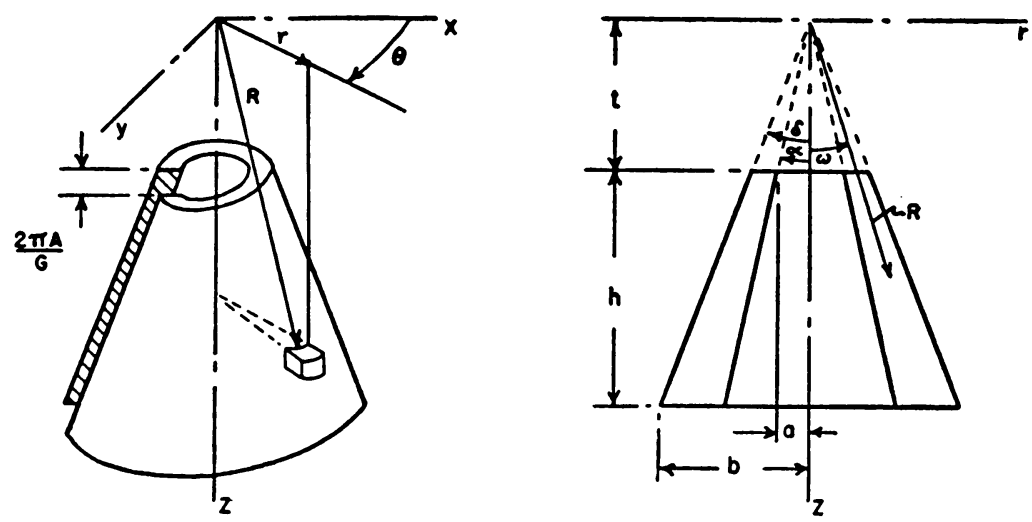

FIG. 3. The hollow cone with a dislocation of order 3 .

for convenience instead of the cylindrical co-ordinates $r, \theta, z$. An element at a conical surface (Fig. 4) has the cylindrical shear component $\tau_{z \theta}$ of (6) as the only non-zero stress in cylindrical co-ordinates, and in spherical co-ordinates this gives rise to two components $\tau_{\theta \omega}$ and $\tau_{\theta R}$, normal and tangential to the conical surface. The normal component $\tau_{0 \omega}$ is shown in Fig. 4, together with its complementary shear $\tau_{\omega \theta}$ on the
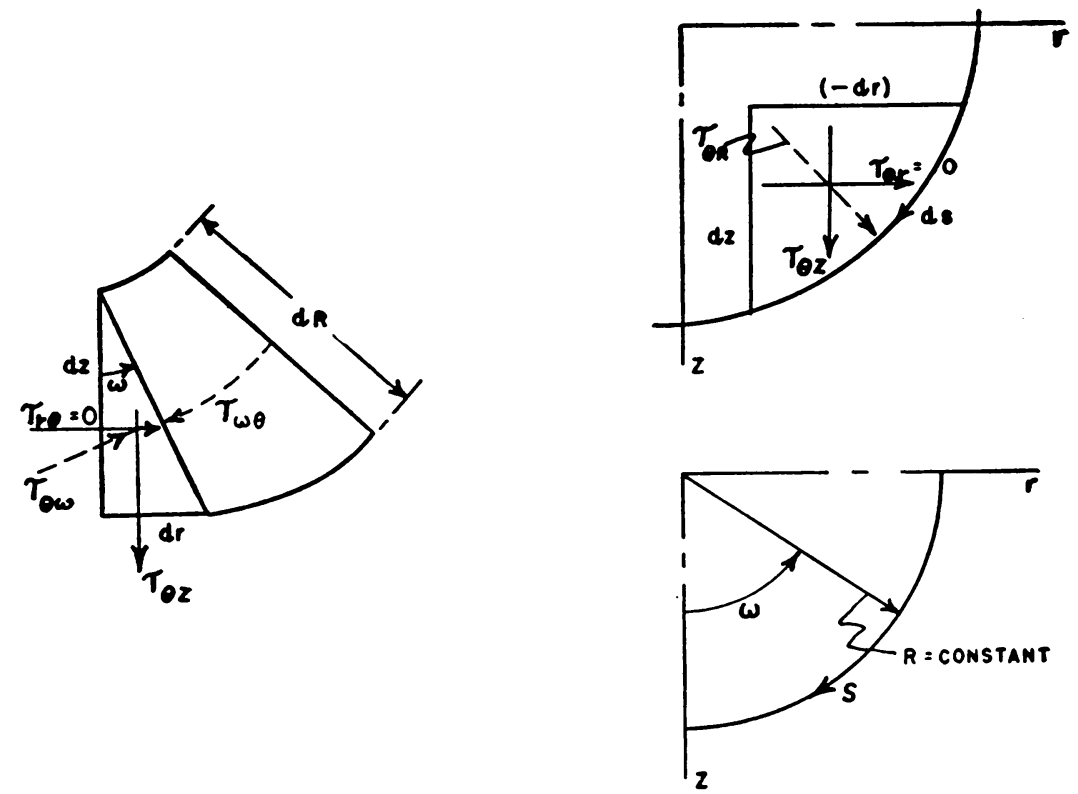

FIg. 4. Transition from erlindrical to spherical co-ordinates and stress components.

conical surface - the ringwise shear which must be removed from the conical boundary surfaces $\omega=\alpha$ and $\omega=\delta$. We have, from Fig. 4 and Eq. 6

$$
\tau_{\theta \omega}=\tau_{\omega \theta}=-\tau_{\theta s} \sin \omega=-\frac{A}{r} \sin \omega=-\frac{A}{R} .
$$


In spherical co-ordinates Michell's equations (10) yield

$$
\tau_{\omega \theta}=-\frac{1}{r^{2}} \frac{\partial \psi}{\partial R}=-\frac{1}{R^{2} \sin ^{2} \omega} \frac{\partial \psi}{\partial R}
$$

and the differential equation (11) for $\psi$ becomes, writing $\mu=\cos \omega$

$$
\frac{\partial}{\partial R} \frac{1}{R^{2}} \frac{1}{\left(1-\mu^{2}\right)^{2}} \frac{\partial \psi}{\partial R}+\frac{\partial}{\partial \mu} \frac{1}{R^{4}} \frac{1}{\left(1-\mu^{2}\right)} \frac{\partial \psi}{\partial \mu}=0 .
$$

In view of (14) and (13) we require a solution of this having a derivative $\partial \psi / \partial R$ which behaves like $R$ on the surfaces $\omega=\alpha$ and $\omega=\delta$. The required form [2] is

$$
\psi=R^{2}\left[B\left(1+\mu^{2}\right)+C \mu\right],
$$

with $B$ and $C$ arbitrary constants.

Combining the state represented by (16) with the state represented by (5), (6), and (7) we find that the stress component $\tau_{\omega \theta}$ is given by

$$
\tau_{\omega \theta}=-\frac{A}{R}-\frac{2 B}{R}\left(1+2 \cot ^{2} \omega\right)-\frac{2 C}{R} \frac{\cot \omega}{\sin \omega} .
$$

For freedom of the conical surfaces this should vanish when $\omega=\alpha$ and when $\omega=\delta$, and therefore $B$ and $C$ are determined as

$$
\begin{aligned}
& B=-\frac{1}{2} A H\left(\sin ^{2} \alpha \cos \delta-\sin ^{2} \delta \cos \alpha\right), \\
& C=-\frac{1}{2} A H\left[\left(1+\cos ^{2} \alpha\right) \sin ^{2} \delta-\left(1+\cos ^{2} \delta\right) \sin ^{2} \alpha\right],
\end{aligned}
$$

where $H=\left[\left(1+\cos ^{2} \alpha\right) \cos \delta-\left(1+\cos ^{2} \delta\right) \cos \alpha\right]^{-1}$. The constant $A$ is determined from (7) by the prescribed displacement discontinuity.

The state of stress so found is conveniently stated in cylindrical co-ordinates as

$$
\begin{aligned}
\tau_{\theta z} & =(A+2 B) r^{-1}+C z r^{-1}\left(r^{2}+z^{2}\right)^{-1 / 2}, \\
\tau_{r \theta} & =-4 B z r^{-2}-C r^{-2}\left(r^{2}+2 z^{2}\right)\left(r^{2}+z^{2}\right)^{-1 / 2}, \\
\sigma_{r} & =\sigma_{\theta}=\sigma_{z}=\tau_{z r}=0 .
\end{aligned}
$$

The condition of zero torque on the ends has not been imposed. Evaluation of the torque from $\tau_{\theta_{z}}$ in (18) shows it to be zero however, and therefore (18) represents the desired solution for the dislocation of order 3 in the hollow cone. Shear corresponding to $\tau_{0,}$ remains as a self-equilibrating distribution on each end, and its removal would alter the stress near thr ends.

3. The hollow sphere. The hollow sphere, or spherical frustum, shown in Fig. 5, is taken first as part of a hollow cylinder in the state expressed by (5), (6), and (7). The shear stress $\tau_{\theta R}$ or $\tau_{R \theta}$, in spherical co-ordinates $R, \omega, \theta$, is (Fig. 4)

$$
\tau_{R \theta}=-\tau_{\theta z} \frac{d r}{d s}=-\frac{A}{r} \frac{d r}{d s} .
$$

For a spherical surface of radius $R, d r / d s=-z / R$. On the boundary surfaces $R=a$, $R=b$ we have therefore

$$
\left(\tau_{R \theta}\right)_{R=a}=\frac{A}{a}\left(\frac{z}{r}\right)_{R=a} ; \quad\left(\tau_{r \theta}\right)_{R=b}=\frac{A}{b}\left(\frac{z}{r}\right)_{R=b} .
$$



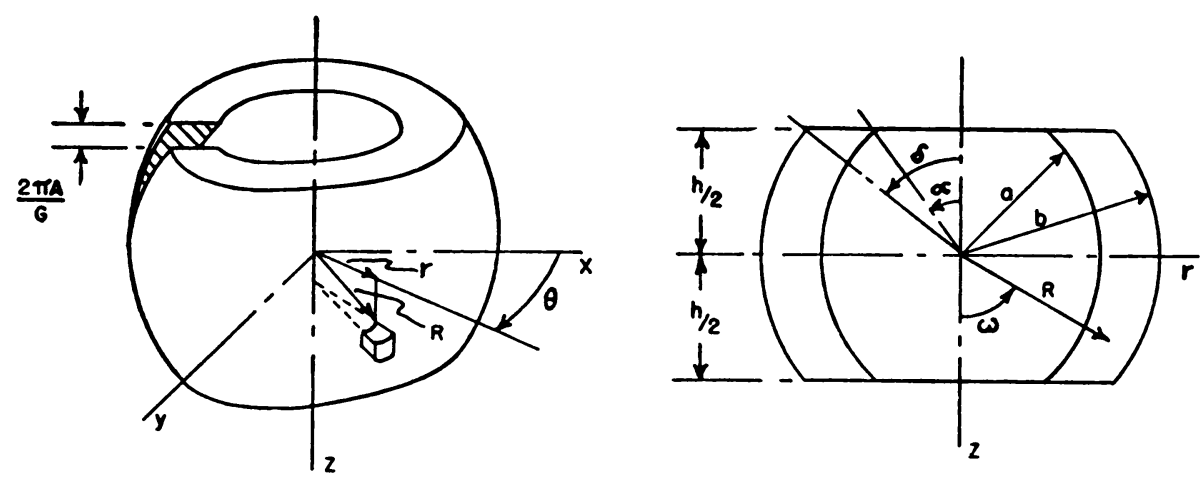

FIG. 5.

To remove these we introduce a Michell function $\psi$ according to (11) and (10). In spherical co-ordinates this leads, by (12), to

$$
\tau_{R \theta}=-\frac{1}{r^{2}}\left(\frac{\partial \psi}{\partial s}\right)_{R}
$$

and so, in view of (19), the boundary conditions on $\psi$ are

$$
\left(\frac{\partial \psi}{\partial s}\right)_{R=a}=A a \sin \omega \cos \omega, \quad\left(\frac{\partial \psi}{\partial s}\right)_{R=b}=A b \sin \omega \cos \omega .
$$

On a surface $R=$ constant we have $d s=-R d \omega$, and with $\mu=\cos \omega$ we may write for this surface

$$
\frac{\partial \psi}{\partial s}=-\frac{1}{R} \frac{\partial \psi}{\partial \omega}=-\frac{1}{R} \frac{\partial \psi}{\partial \mu} \frac{d \mu}{d \omega}=\frac{1}{R} \sin \omega \frac{\partial \psi}{\partial \mu} .
$$

Introducing this in (20) yields

$$
\left(\frac{\partial \psi}{\partial \mu}\right)_{R=a}=A a^{2} \mu, \quad\left(\frac{\partial \psi}{\partial \mu}\right)_{R=b}=A b^{2} \mu .
$$

The solution of (15) appropriate to these conditions is the $B$ solution from (16),

$$
\psi=B R^{2}\left(1+\mu^{2}\right)
$$

and both conditions (21) are satisfied if $B=A / 2$.

Superposition of the stress represented by this function, and the stress (6), gives the following state of stress in cylindrical co-ordinates

$$
\begin{aligned}
\tau_{\theta z} & =2 A / r, \quad \tau_{r \theta}=-2 A z / r, \\
\sigma_{r} & =\sigma_{\theta}=\sigma_{z}=\tau_{z r}=0 .
\end{aligned}
$$

The torque on the ends of the hollow spherical frustum (Fig. 5) is

$$
T=\int_{\operatorname{ain} \alpha}^{b \sin \delta} \tau_{\theta z} 2 \pi r^{2} d r=2 \pi A\left(b^{2} \sin ^{2} \delta-a^{2} \sin ^{2} \alpha\right) .
$$

It may be removed by means of the $\psi$-function corresponding to torsion of a sphere

$$
\psi=F R^{3} \quad(F \text { a constant }) .
$$


On a spherical surface $R=$ constant this function is constant, and the surface is consequently free. The stress and torque are (reverting to cylindrical co-ordinates)

$$
\begin{gathered}
\tau_{\theta z}=3 F r^{-1}\left(r^{2}+z^{2}\right)^{1 / 2}, \quad \tau_{r \theta}=-3 F z r^{-2}\left(r^{2}+z^{2}\right)^{1 / 2}, \\
T=\int_{a \sin \alpha}^{b \sin \delta} \tau_{\theta z} 2 \pi r^{2} d r=2 \pi F\left(b^{3}-a^{3}\right) .
\end{gathered}
$$

Combining this with (22), (23), vanishing of the total torque requires $F=-A\left(b^{2} \sin ^{2} \delta-\right.$ $\left.a^{2} \sin ^{2} \alpha\right)\left(b^{3}-a^{3}\right)^{-1}$, and we find as the solution for the dislocation of order 3 for the hollow spherical frustum, corresponding to the displacement discontinuity (7),

$$
\begin{aligned}
\tau_{\theta z} & =A\left[2 r^{-1}-3 r^{-1}\left(r^{2}+z^{2}\right)^{1 / 2}\left(b^{2} \sin ^{2} \delta-a^{2} \sin ^{2} \alpha\right)\left(b^{3}-a^{3}\right)^{-1}\right], \\
\tau_{r \theta} & =A\left[-2 z r^{-2}+3 z r^{-2}\left(r^{2}+z^{2}\right)^{1 / 2}\left(b^{2} \sin ^{2} \delta-a^{2} \sin ^{2} \alpha\right)\left(b^{3}-a^{3}\right)^{-1}\right], \\
\sigma_{r} & =\sigma_{\theta}=\sigma_{z}=\tau_{z r}=0 .
\end{aligned}
$$

Again a self-equilibrating distribution of $\tau_{\theta z}$ remains on the ends.

All these dislocations, for cylinder, cone, and sphere, have singularities in the stress on the axis $r=0$, necessitating the restriction to the hollow cylinder and the frustum of the cone or sphere.

Ghosh [3] has given the dislocations of orders 2 and 6 (Fig. 1) for the hollow sphere. The result (25) adds a third to this set.

\section{REFERENCES}

1. V. Volterra, Sur l'equilibre des corps élastiques multiplement connexés, Ann. Sci. de l'Ecole Normale Supérieure, Ser. 3, 24, 401 (1907)

2. J. C. Wilhoit, Jr., An addition to Poritsky's solution of a differential equation of torsion, Quart. Appl. Math. 11, 499 (1954)

3. S. Ghosh, On some many-valued solutions of the equations of elastic equilibrium in polar co-ordinates, Z. angew. Math. u. Mech. 12, 188 (1932) 